\title{
Chemical composition mapping with nanometre resolution by soft X-ray microscopy
}

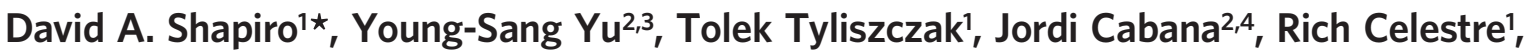 \\ Weilun Chao5, Konstantin Kaznatcheev ${ }^{6}$, A. L. David Kilcoyne', Filipe Maia7, Stefano Marchesini, \\ Y. Shirley Meng ${ }^{3}$, Tony Warwick ${ }^{1}$, Lee Lisheng Yang ${ }^{1}$ and Howard A. Padmore ${ }^{1}$
}

$\mathrm{X}$-ray microscopy is powerful in that it can probe large volumes of material at high spatial resolution with exquisite chemical, electronic and bond orientation contrast ${ }^{1-5}$. The development of diffraction-based methods such as ptychography has, in principle, removed the resolution limit imposed by the characteristics of the X-ray optics ${ }^{6-10}$. Here, using soft X-ray ptychography, we demonstrate the highest-resolution $X$-ray microscopy ever achieved by imaging $5 \mathrm{~nm}$ structures. We quantify the performance of our microscope and apply the method to the study of delithiation in a nanoplate of $\mathrm{LiFePO}_{4}$, a material of broad interest in electrochemical energy storage ${ }^{11,12}$. We calculate chemical component distributions using the full complex refractive index and demonstrate enhanced contrast, which elucidates a strong correlation between structural defects and chemical phase propagation. The ability to visualize the coupling of the kinetics of a phase transformation with the mechanical consequences is critical to designing materials with ultimate durability.

Ptychography uses coherent diffraction patterns recorded from fields of illumination that are physically overlapped on an extended sample, with known separation. This information is used in the iterative reconstruction of phase, and results in a robust and unique solution that gives the local complex refractive index of the object and the complex illumination function ${ }^{13}$. Through phase retrieval from diffraction data, ptychography can in principle overcome the limitations of X-ray optics, namely limited efficiency and numerical aperture. Ptychographic imaging at high spatial resolution requires the data redundancy provided by overlapping X-ray probe positions, a good signal-to-noise ratio in the diffraction data, a high-performance scanning system, illumination with stability commensurate with the desired spatial resolution, and algorithms for fast and precise reconstruction of the data. However, the spatial resolution of ptychographic microscopes has, until this work, not surpassed that of the best conventional systems. The method has been largely developed on hard X-ray sources and using simple pinhole optics for illumination, resulting in a low scattering cross-section or low coherent intensity at the sample; accordingly, exposure times have been long and the needs for high mechanical and illumination stability exacerbated. As coherent power scales as $\lambda^{2}$ and the scattering cross-section also scales by approximately the same factor, the use of soft X-rays with wavelengths of $\sim 1-10 \mathrm{~nm}$ is a key enabling aspect for high-resolution microscopy ${ }^{14}$.

In a standard scanning transmission X-ray microscope $(\mathrm{STXM})^{15-17}$, the sample is raster-scanned through the X-ray beam (under interferometric feedback control), while the total transmitted intensity is measured at each position, thus yielding a map of the material optical density at the resolution of the X-ray focus size. In a soft X-ray STXM, imaging with a $25 \mathrm{~nm}$ focusing optic can proceed with exposure times of $<1 \mathrm{~ms}$. In the case of ptychographic imaging, the large dynamic range needed for high-resolution diffraction measurements (which scales as the focus size divided by the resolution element to the fourth power) is achieved by a dual exposure mode that merges long and short exposure diffraction measurements at each probe position (see Supplementary Information, page 2). Throughout the ptychography scan, we maintain the sample and focusing optic in relative alignment using an interferometric feedback system with a precision comparable to the wavelength of the X-ray illumination (Fig. 1). Scattering from the interferometer laser optics and other X-ray sources can lead to

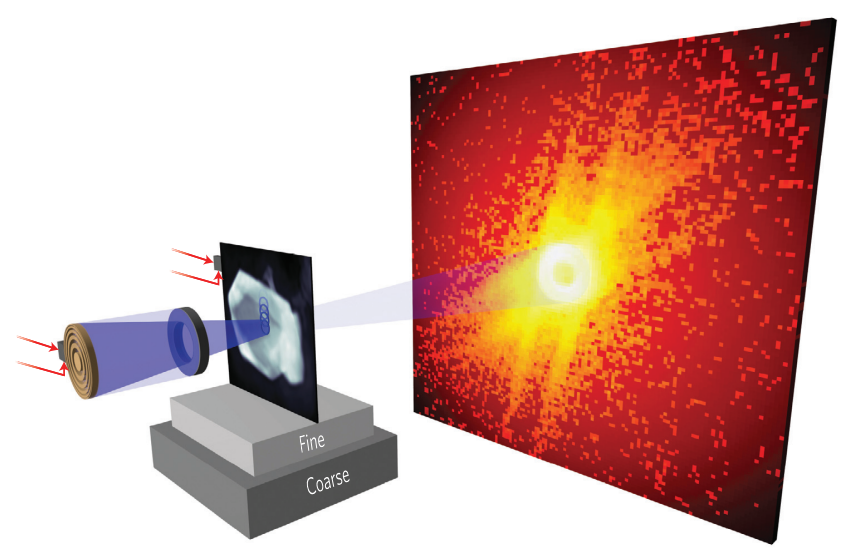

Figure 1 | Layout of the soft X-ray ptychographic microscope. A $60 \mathrm{~nm}$ outer-zone-width zone plate focuses a coherent soft $X$-ray beam onto the sample, which is scanned in $40 \mathrm{~nm}$ increments to ensure overlap of the probed areas. An order-sorting aperture blocks all but the first-order focus from the zone plate optic. Diffraction data are recorded on an X-ray CCD located $80 \mathrm{~mm}$ downstream of the sample, while the sample position is stabilized with respect to the zone plate by an interferometer (red arrows) that measures relative displacements in the scanning directions with $1.5 \mathrm{~nm}$ resolution. The central annulus in the diffraction pattern is the band-limited diffraction pattern of the X-ray probe, and all photons scattered outside this annulus contribute to the increased spatial resolution of the imaging system.

\footnotetext{
'Advanced Light Source, Lawrence Berkeley National Laboratory, Berkeley, California 94720, USA, ${ }^{2}$ Environmental Energy Technologies Division, Lawrence Berkeley National Laboratory, Berkeley, California 94720, USA, ${ }^{3}$ Department of NanoEngineering, University of California at San Diego, San Diego, California 92093, USA, ${ }^{4}$ Department of Chemistry, University of Illinois at Chicago, Chicago, Illinois 60607, USA, ${ }^{5}$ Center for X-ray Optics, Lawrence Berkeley National Laboratory, Berkeley, California 94720, USA, ${ }^{6}$ NSLS-II, Brookhaven National Laboratory, Upton, New York 11973, USA, ${ }^{1}$ Laboratory of Molecular Biophysics, Uppsala University, SE-751 24 Uppsala, Sweden. *e-mail: dashapiro@lbl.gov
} 
a

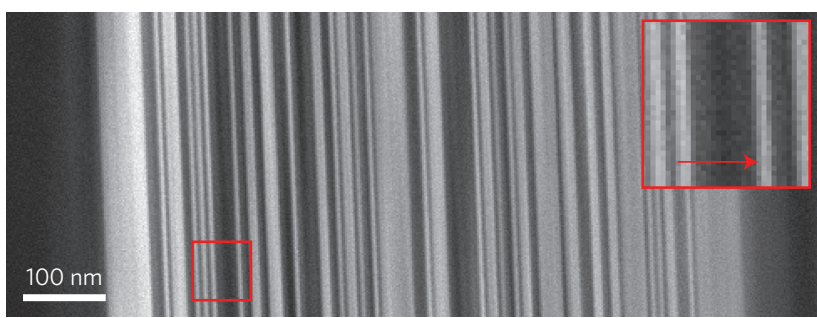

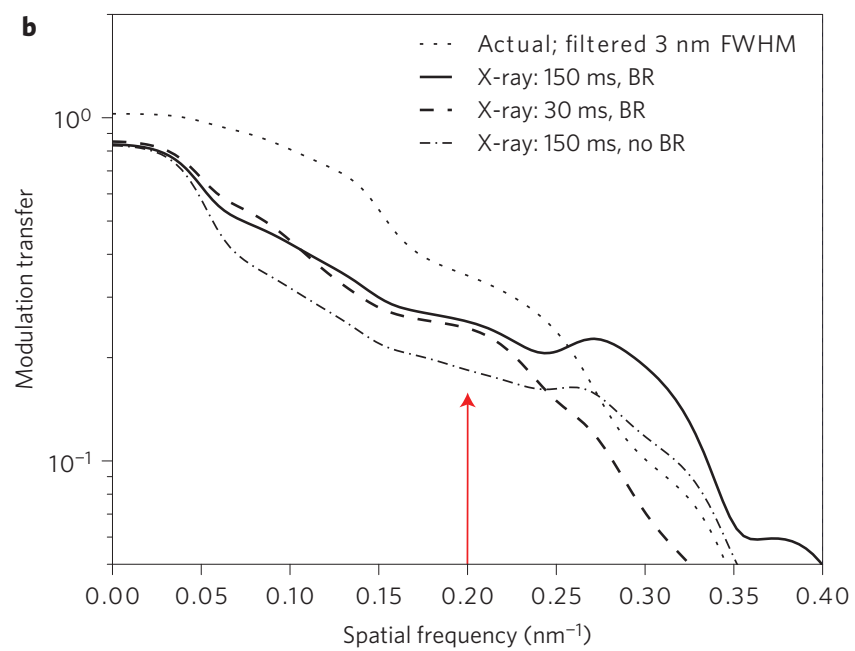

Figure 2 | Ptychographic reconstruction of a resolution test object. a, Phase of the ptychographic reconstruction of the multilayer test object. The finest half-period line width of $5 \mathrm{~nm}$ is clearly resolved. Pixel size, $2 \mathrm{~nm}$. Scale bar, $100 \mathrm{~nm}$. The red arrow in the inset image points to a single line of $5 \mathrm{~nm}$ width. b. Modulation transfer of the filtered actual sequence and the $\mathrm{X}$-ray phase images with different exposure times and with and without background refinement (BR). The MTF is calculated as the square root of the ratio of the X-ray PSD to the actual PSD (smoothed by a Gaussian with 5 pixel (r.m.s.) width). The PSDs for the actual sequences are calculated from a single line, while that of the X-ray images is the average of all lines in the image (Supplementary Figs 1 and 2). The MTF shows 25\% modulation transfer at $5 \mathrm{~nm}$ half-period (red arrow) and reaches the Rayleigh resolution, or 11\% contrast, at $3 \mathrm{~nm}$ half-period.
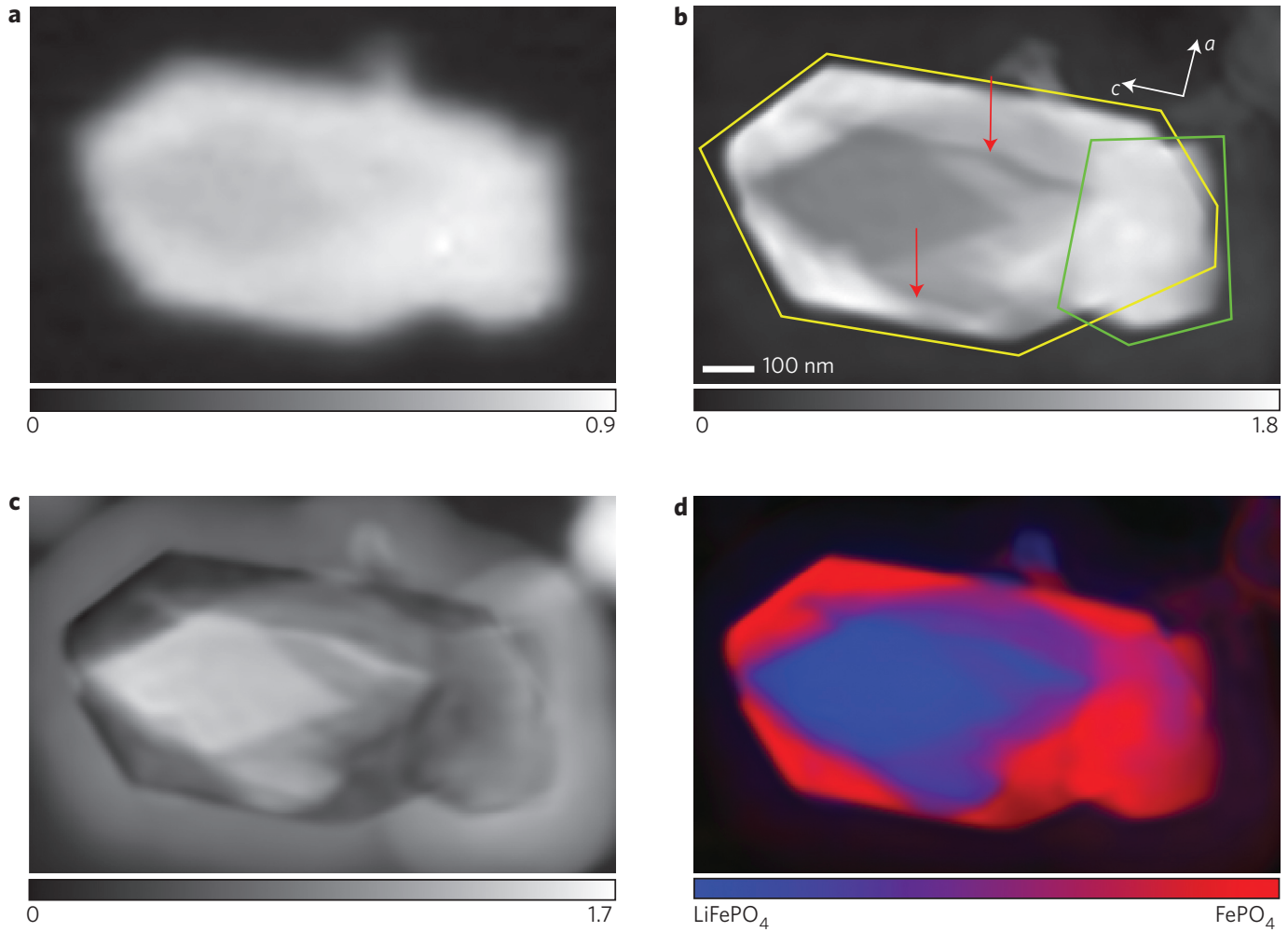

Figure 3 | X-ray microscopy of partially delithiated LiFePO 4 . a,b, Optical density maps from STXM (a) and ptychography (b) at 710 eV, showing maximum absorption contrast between the end members. The spatial resolution of the STXM is not adequate to visualize the presence of multiple particles, which are outlined in the ptychographic image, and cracks along the crystallographic $c$ axis, which are indicated by red arrows (the $a$ and $c$ crystallographic axes are indicated by white arrows; the $b$ axis is parallel to the X-ray transmission). The crack is only on the surface and reveals an unreacted domain below. Furthermore, the ptychographic image demonstrates a mismatch between the apex of the unreacted phase and that of the crystal lattice, and an asymmetry in the unreacted domain. Pixel size, $4.0 \mathrm{~nm}$. Scale bar, $100 \mathrm{~nm}$. c, Phase of the ptychographic reconstruction at $709.2 \mathrm{eV}$, showing maximum relative phase shift between the end members. The halo around the particle is carbon contamination, which builds up with X-ray exposure and is not apparent in the absorption images. d, Colourized composition map calculated by principal component analysis, clustering and singular value decomposition of the full complex refractive index. Only material containing the end members is apparent. The spectra used for the fitting are shown in Fig. 4. 

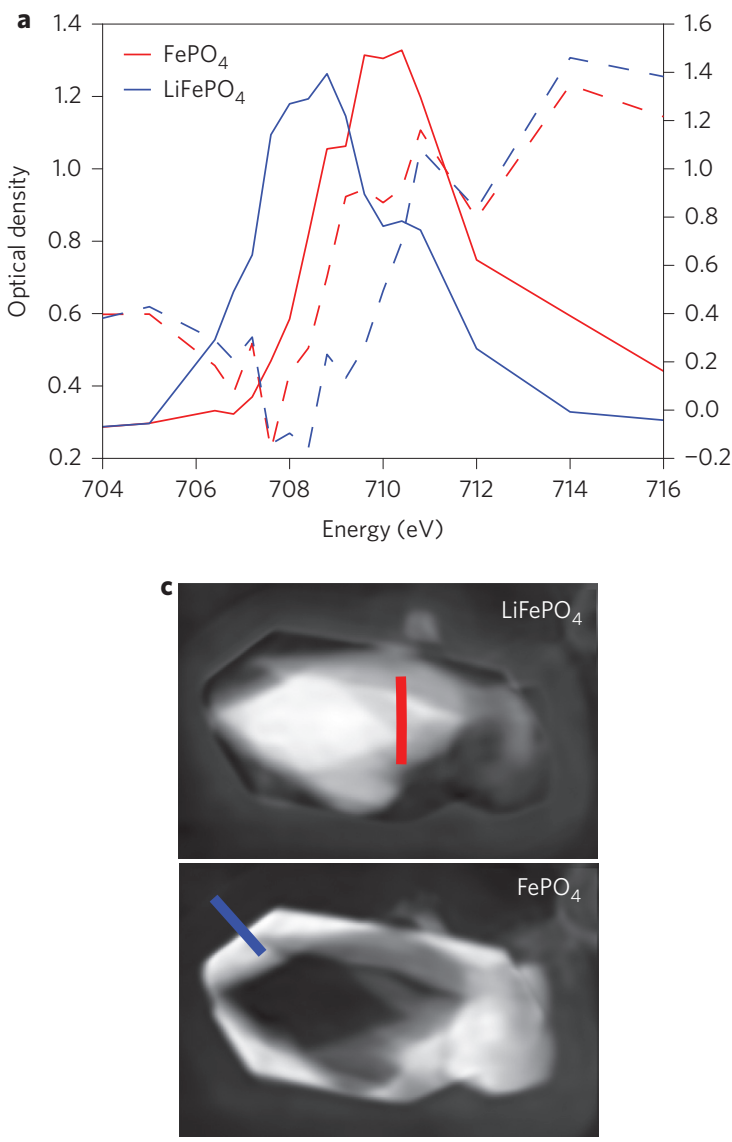
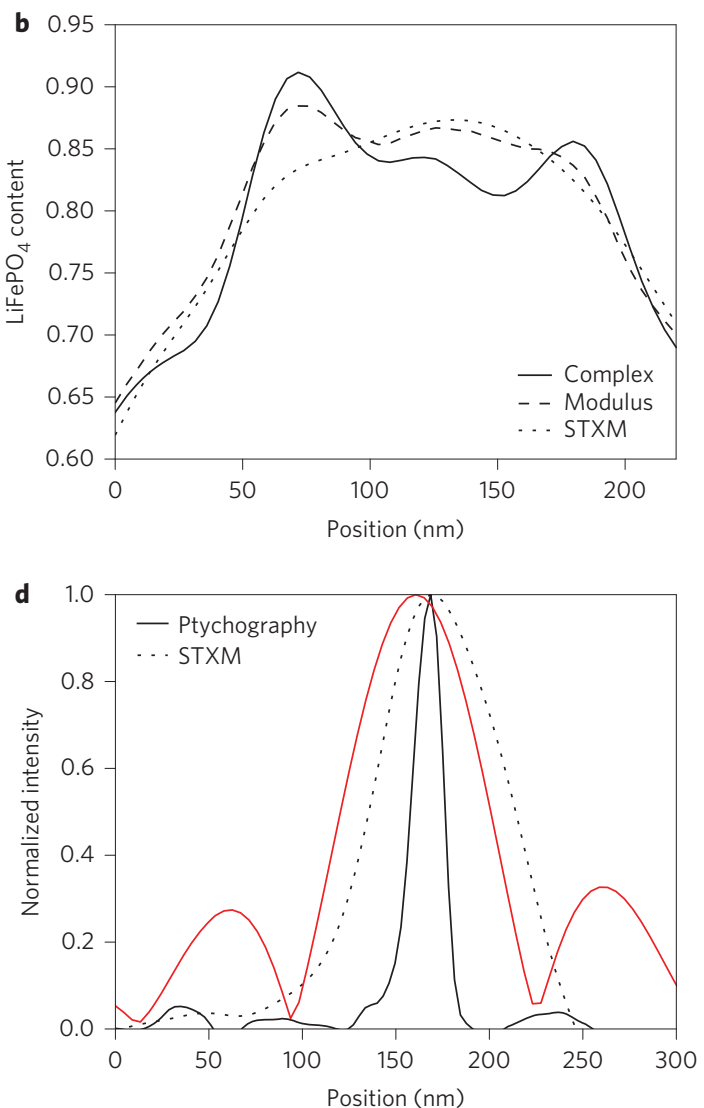

Figure 4 | Results of chemical mapping. a, Absorption (solid lines) and phase spectra (dashed lines) calculated from the singular value decomposition and used for composition mapping. The maximum relative phase shift occurs at an X-ray energy (709.2 eV) about $0.8 \mathrm{eV}$ lower than that for maximum absorption contrast $(710 \mathrm{eV})$. b, Line-outs across the $\mathrm{LiFePO}_{4}$ component map (straight red line in c), which shows enhanced chemical contrast when calculated using the full complex refractive index when compared with distributions calculated just from the modulus of the reconstructions. c, Chemical component maps for the two end members. d, Derivatives of line-outs across the edge of the FePO 4 component map (straight blue line in c), which give estimates of the spatial resolution. The FWHM is $18 \mathrm{~nm}$ for the ptychographic chemical map and $70 \mathrm{~nm}$ for STXM with a $25 \mathrm{~nm}$ optic. For comparison, a slice through the X-ray probe, reconstructed from the ptychography data, is shown (red curve) which has a FWHM of $150 \mathrm{~nm}$ from a $60 \mathrm{~nm}$ optic because of the strong tails.

an incoherent background signal, which cannot be measured readily. We eliminate the effect of all incoherent background signals through the implementation of a background retrieval algorithm, which iteratively determines a constant offset to the diffraction data (constant across frames but varying from pixel to pixel) by solving an offset minimization problem ${ }^{18}$ (Supplementary Fig. 1).

To demonstrate the technique and quantify the spatial resolution of the system, we have imaged a pseudo random binary test pattern ${ }^{19}$. The structure is designed to have a flat power spectrum out to the minimum half-period line width of $5 \mathrm{~nm}$. A ptychographic phase contrast image of the test structure is presented in Fig. 2, which shows that the $5 \mathrm{~nm}$ lines are clearly resolved. A modulation transfer function (MTF) of the system can be defined as the ratio of the square root of the power spectral density (PSD) (Supplementary Figs 1 and 2) of the microscope image to that of the actual sequence ${ }^{20}$, determined by high-resolution scanning electron microscopy (SEM). This function is plotted for several cases in Fig. 2b. The optimal case shows a modulation of $25 \%$ at $5 \mathrm{~nm}$ halfperiod and a Rayleigh resolution given by $11 \%$ modulation at $3 \mathrm{~nm}$. This can be compared to the resolution defined by the wavelength and the numerical aperture limit of the detector of $2.7 \mathrm{~nm}$. The MTF clearly shows the improved resolution that comes with additional X-ray exposure, a characteristic unique to diffractionbased imaging schemes. Furthermore, we find enhanced contrast across the full spectrum with the application of the background refinement algorithm.

As a demonstration of the dramatic improvement in resolution and chemical speciation that can be provided over standard STXM by a ptychographic microscope, we have studied the mechanism of delithiation in a single nanoplate of $\mathrm{LiFePO}_{4}$, a compound that has become a canonical electrode material in the study of electrochemical energy storage ${ }^{21}$. It is now established that particle dimensions well below $1 \mu \mathrm{m}$ are required for $\mathrm{LiFePO}_{4}$ electrodes to function. However, extensive efforts are still directed towards relating its macroscopic properties, especially its extremely fast kinetics at such small sizes ${ }^{11}$, with the (de)lithiation reaction mechan$i^{22}$. A significant barrier to effectively studying this mechanism at the single-crystal level is the limited number of tools available that offer sufficient chemical and spatial resolution for the visualization of compound distribution in sub-micrometre particles ${ }^{23,24}$.

Figure 3 presents a comparison of images obtained in both normal STXM mode (Fig. 3a), using a $25 \mathrm{~nm}$ optic, and in ptychographic mode (Fig. 3b), using a $60 \mathrm{~nm}$ optic. The ptychographic images show a maximum chemical contrast between $\mathrm{LiFePO}_{4}$ and $\mathrm{FePO}_{4}$ at $710 \mathrm{eV}$ for absorption and $709.2 \mathrm{eV}$ for relative phase shift. These images, unlike the STXM image, both show a clear internal chemical domain pattern, while the increased sensitivity of the phase image reveals a halo of carbon contamination that 
builds up with X-ray exposure. Chemical distributions can be calculated from a sequence of images recorded at different incident $\mathrm{X}$-ray energies spanning an atomic resonance, which provides a spectrum at each point in the sample. Such distributions typically proceed from absorption spectra ${ }^{25}$; however, ptychography provides the full complex refractive index. We record images across the $\mathrm{Fe}$ L-edge and use these as input to principal component analysis, followed by $k$-means clustering and finally singular value decomposition providing the complex-valued spectra (Fig. 4a). The resulting composition map shows clear definition of the two chemical species without carbon contamination (Fig. 3d) and higher chemical specificity than maps calculated from the absorption alone (Fig. 4b). Furthermore, the spatial resolution of the chemical distribution maps (Fig. 4c), at $18 \mathrm{~nm}$, is nearly an order of magnitude better than the probe full-width at half-maximum (FWHM) of $150 \mathrm{~nm}$ and more than three times better than state-of-the-art STXM (Fig. 4d, Supplementary Figs 1-3). The absorption spectra obtained from these images agree with standards for pure $\mathrm{LiFePO}_{4}$ and $\mathrm{FePO}_{4}$ (Supplementary Fig. 2), confirming that (1) the reaction is probably two-phase and (2) both phases can coexist, even in these small plates. The cluster analysis was carried out assuming the presence of only two phases; however, the presence of a third phase at low concentration cannot be excluded.

Our enhanced spatial resolution and chemical specificity provide critical new insights. There is an identifiable correspondence between the formation of two cracks along the $c$-direction, on opposite sides of the crystal (Fig. 3b,c), and the phase transformation. Surface cracking is apparent in SEM images collected for other crystals in the same sample (Supplementary Figs 1-4) and is expected because of lattice shrinkage along the $a$-axis during the $\mathrm{LiFePO}_{4}$ $\mathrm{FePO}_{4}$ transition ${ }^{23,26}$. Significant delithiation is found to occur on both sides of each crack (marked by arrows in Fig. 3b), but little reaction is observed within them as the $\mathrm{LiFePO}_{4}$ content is greater than $90 \%$ (Fig. 4b). This indicates that the crack formation exposes fresh unreacted domains under the surface and therefore that delithiation is more rapid along the $a-c$ plane than in the $b$-direction, meaning that the reaction is solid-state-limited. It follows that the complexity of the distribution of phases is probably the result of the reaction proceeding under the kinetic control imposed by microstructural defects, which are present in the pristine crystals (see small pits and grooves in Supplementary Fig. 2) and develop as a result of stress during the reaction.

It should be noted that the X-ray source used for the chemical mapping was a bending magnet and will be superseded by a new optimized undulator source that will be a factor of $10^{3}-10^{4}$ times brighter. When coupled to new high-frame-rate charge-coupled device (CCD) sensors ${ }^{27}$, this will enable exposure times of a few milliseconds and a spatial resolution at the wavelength of the radiation. Beyond this, a new generation of diffraction-limited storage rings will increase the coherent power by a further factor of $100-1,000$, opening up the possibility of wavelength-limitedresolution tomography. The ability to record chemically sensitive maps with few-nanometre spatial resolution is already a revolutionary step forward. The added phase information that the method provides will enable the high-resolution study of systems where the absorption contrast is low or where radiation damage is a concern, such as with polymers and metal-organic frameworks. Finally, wavelength-limited spatial resolution will enable the study of chemical phases and transport at the scale of single nanocrystals and nanopores in hierarchical and porous materials. These capabilities are set to revolutionize the study of chemical phases at the mesoscale.

\section{Methods}

The ptychographic measurements utilized an optic with $60 \mathrm{~nm}$ outer zone width and proceeded in focus with a square scan grid of $40 \mathrm{~nm}$ steps. The resolution test sample was a multilayered structure composed of alternating layers of $\mathrm{Si}$ and $\mathrm{Mo}$ thinned to $200 \mathrm{~nm}$ thickness using focused ion beam milling. Diffraction data were recorded on a Princeton Instruments MTE-1300B in-vacuum CCD camera in a double exposure mode without the use of a beamstop (see Supplementary Information, page 2). Measurements of the test object proceeded on the undulator beamline 11.0.2 using combinations of 30/150 ms exposure times (with $4 \times 10^{7}$ photons s ${ }^{-1}$, total time per point of $1.2 \mathrm{~s}$ ), and measurements of $\mathrm{LiFePO}_{4}$ proceeded on the bending magnet beamline 5.3.2.1 using 30/800 ms exposure times (with $5 \times 10^{5}$ photons $\mathrm{s}^{-1}$, total time per point of $1.8 \mathrm{~s}$ ). The surface dose deposited was $2 \times 10^{12} \mathrm{~Gy}$ for the multilayer sample and $7 \times 10^{10} \mathrm{~Gy}$ for the $\mathrm{LiFePO}_{4}$ particle. This compares well to the doses required for imaging at our measured resolutions, $4 \times 10^{12} \mathrm{~Gy}$ and $16 \times 10^{10} \mathrm{~Gy}$, respectively ${ }^{17}$. The data were reconstructed using 200 iterations of an implementation of the $\mathrm{RAAR}^{28}$ algorithm using an illumination refinement and a background refinement during each iteration, beginning after the tenth iteration ${ }^{18}$. The ptychography algorithms were implemented in CUDA and took $\sim 0.05$ s per iteration $(0.2 \mu$ s per image pixel per iteration) on a 16 GPU (nVidia GTX Titan) cluster for data sets that comprise $\sim 1,000$ diffraction patterns of $128 \times 128$ pixels. The reconstructed images were $600 \times 500$ pixels. $\mathrm{LiFePO}_{4}$ nanoplates of known crystallographic orientation were synthesized in hydrothermal conditions and delithiated to a nominal batch composition of $\mathrm{Li}_{0.55} \mathrm{FePO}_{4}$ using dilute $\mathrm{Br}_{2}$ solutions in acetonitrile, following standard procedures ${ }^{23,29}$. Complete ptychography scans were recorded at fixed energies in $1 \mathrm{eV}$ intervals around the $\mathrm{Fe}$ L-edge, spanning from $700 \mathrm{eV}$ to $716 \mathrm{eV}$, and $0.4 \mathrm{eV}$ intervals near the absorption resonance from $706 \mathrm{eV}$ to $712 \mathrm{eV}$. Chemical maps were generated using an automated procedure of complex-valued principal component analysis followed by expectation-maximization clustering ( $k$-means algorithm), and finally singular value decomposition (see Supplementary Information, page 2).

Received 10 March 2014; accepted 22 July 2014; published online 7 September 2014

\section{References}

1. Kirz, J., Jacobsen, C. \& Howells, M. Soft X-ray microscopes and their biological applications. Q. Rev. Biophys. 28, 33-130 (1995).

2. Ade, H. \& Stoll, H. Near-edge X-ray absorption fine-structure microscopy of organic and magnetic materials. Nature Mater. 8, 281-290 (2009).

3. Chao, W., Kim, J., Rekawa, S., Fischer, P. \& Anderson, E. H. Demonstration of $12 \mathrm{~nm}$ resolution Fresnel zone plate lens based soft X-ray microscopy. Opt. Express 17, 17669-17677 (2009).

4. Rehbein, S., Heim, S., Guttmann, P., Werner, S. \& Schneider, G. Ultrahighresolution soft-X-ray microscopy with zone plates in high orders of diffraction. Phys. Rev. Lett. 103, 110801 (2009).

5. Vila-Comamala, J. et al. Zone-doubled Fresnel zone plates for high-resolution hard X-ray full-field transmission microscopy. J. Synchrotron Radiat. 19, 705-709 (2012).

6. Rodenburg, J. M. et al. Hard-X-ray lensless imaging of extended objects. Phys. Rev. Lett. 98034801 (2007).

7. Thibault, P. et al. High-resolution scanning X-ray diffraction microscopy. Science 321, 379-382 (2008).

8. Dierolf, M. et al. Ptychographic X-ray computed tomography at the nanoscale. Nature 467, 436-439 (2010).

9. Holler, M. et al. An instrument for 3D X-ray nano-imaging. Rev. Sci. Instrum. 83, 073703 (2012).

10. Schropp, A. et al. Hard X-ray scanning microscopy with coherent radiation: beyond the resolution of conventional X-ray microscopes. Appl. Phys. Lett. 100, 253112 (2012).

11. Kang, B. \& Ceder, G. Battery materials for ultrafast charging and discharging. Nature 458, 190-193 (2009).

12. Tarascon, J.-M. Key challenges in future Li-battery research. Phil. Trans. R. Soc. Math. Phys. Eng. Sci. 368, 3227-3241 (2010).

13. Thibault, P., Dierolf, M., Bunk, O., Menzel, A. \& Pfeiffer, F. Probe retrieval in ptychographic coherent diffractive imaging. Ultramicroscopy 109, 338-343 (2009).

14. Howells, M. R. et al. An assessment of the resolution limitation due to radiationdamage in X-ray diffraction microscopy. J. Electron Spectrosc. Relat. Phenom. 170, 4-12 (2009).

15. Kilcoyne, D. et al. A new scanning transmission X-ray microscope at the ALS for operation up to $2500 \mathrm{eV}$. AIP Conference Proceedings Vol 1234, 465-468 (2010).

16. Bluhm, H. et al. Soft X-ray microscopy and spectroscopy at the molecular environmental science beamline at the Advanced Light Source. J. Electron Spectrosc. Relat. Phenom. 150, 86-104 (2006).

17. Kilcoyne, A. L. D. et al. Interferometer-controlled scanning transmission $\mathrm{X}$-ray microscopes at the Advanced Light Source. J. Synchrotron Radiat. 10, 125-136 (2003).

18. Marchesini, S., Schirotzek, A., Yang, C., Wu, H. \& Maia, F. Augmented projections for ptychographic imaging. Inverse Problems 29, 115009 (2013).

19. Yashchuk, V. V. et al. Characterization of electron microscopes with binary pseudo-random multilayer test samples. Nucl. Instrum. Methods Phys. Res. A 649, 150-152 (2011) 
20. Jacobsen, C. et al. Diffraction-limited imaging in a scanning transmission X-ray microscope. Opt. Commun. 86, 351-364 (1991).

21. Yuan, L.-X. et al. Development and challenges of $\mathrm{LiFePO}_{4}$ cathode material for lithium-ion batteries. Energy Environ. Sci. 4, 269-284 (2011).

22. Malik, R., Aziz, A. \& Ceder, G. A critical review of the Li insertion mechanisms in $\mathrm{LiFePO}_{4}$ electrodes. J. Electrochem. Soc. 160, A3179-A3197 (2013).

23. Boesenberg, U. et al. Mesoscale phase distribution in single particles of $\mathrm{LiFePO}_{4}$ following lithium deintercalation. Chem. Mater. 25, 1664-1672 (2013).

24. Moreau, P., Mauchamp, V., Pailloux, F. \& Boucher, F. Fast determination of phases in $\mathrm{Li}_{x} \mathrm{FePO}_{4}$ using low losses in electron energy-loss spectroscopy. Appl. Phys. Lett. 94, 123111 (2009).

25. Lerotic, M., Jacobsen, C., Schafer, T. \& Vogt, S. Cluster analysis of soft X-ray spectromicroscopy data. Ultramicroscopy 100, 35-37 (2004).

26. Chen, G., Song, X. \& Richardson, T. J. Electron microscopy study of the $\mathrm{LiFePO}_{4}$ to $\mathrm{FePO}_{4}$ phase transition. Electrochem. Solid-State Lett. 9, A295-A298 (2006).

27. Denes, P., Doering, D., Padmore, H. A., Walder, J.-P. \& Weizeorick, J. A fast, direct X-ray detection charge-coupled device. Rev. Sci. Instrum. 80, 083302 (2009).

28. Luke, D. R. Relaxed averaged alternating reflections for diffraction imaging. Inverse Problems 21, 37-50 (2005)

29. Dokko, K., Koizumi, S., Nakano, H. \& Kanamura, K. Particle morphology, crystal orientation, and electrochemical reactivity of $\mathrm{LiFePO}_{4}$ synthesized by the hydrothermal method at 443 K. J. Mater. Chem. 17, 4803-4810 (2007).

\section{Acknowledgements}

All measurements were carried out at either beamline 11.0.2 or beamline 5.3.2.1 at the Advanced Light Source (ALS). The ALS is supported by the Director, Office of Science, Office of Basic Energy Sciences, of the US Department of Energy (contract no.
DE-AC02-05CH11231). The authors acknowledge the support of ALS technical and safety staff and discussions with J. Kirz and J. Spence. This work is partially supported by the Center for Applied Mathematics for Energy Research Applications (CAMERA), which is a partnership between Basic Energy Sciences (BES) and Advanced Scientific Computing Research (ASRC) at the US Department of Energy. The chemical imaging work on $\mathrm{LiFePO}_{4}$ carried out by Y.S.Y., J.C. and Y.S.M. was supported as part of the Northeastern Center for Chemical Energy Storage, an Energy Frontier Research Center funded by the US Department of Energy, Office of Science, Office of Basic Energy Sciences (award no. DE-SC0001294). The authors thank G. Chen (LBNL) for supplying the delithiated $\mathrm{LiFePO}_{4}$ sample. J.C. thanks T. Richardson and R. Kostecki (LBNL) for technical discussions.

\section{Author contributions}

D.A.S., S.M., T.T., K.K., H.P., T.W. and J.C. conceived and planned the experiment. D.A.S., S.M., T.T., R.C., A.S., D.K., T.W., W.C. and L.Y. developed experimental techniques, software and equipment. W.C. and Y.S.Y. prepared the samples. D.A.S., T.T., K.K., D.K. and Y.S.Y. carried out the measurements. D.A.S., S.M. and F.M. developed data processing and ptychography reconstruction codes. D.A.S. and Y.S.Y. performed post-experiment data analysis and Y.S.Y., Y.S.M. and J.C. established the interpretation of the chemical maps. D.A.S., Y.S.Y. and J.C. prepared the manuscript, which incorporates input from all authors.

\section{Additional information}

Supplementary information is available in the online version of the paper. Reprints and permissions information is available online at www.nature.com/reprints. Correspondence and requests for materials should be addressed to D.A.S.

\section{Competing financial interests}

The authors declare no competing financial interests. 